\title{
EWS-Fli1 Antisense Oligodeoxynucleotide Inhibits Proliferation of Human Ewing's Sarcoma and Primitive Neuroectodermal Tumor Cells
}

\author{
Kazuhiro Tanaka, Tomoo Iwakuma, Katsumi Harimaya, Hideshi Sato, and Yukihide Iwamoto \\ Department of Orthopaedic Surgery, Faculty of Medicine, Kyushu University, Fukuoka 812, Japan
}

\begin{abstract}
The translocation $\mathrm{t}(11 ; 22)$ is a common chromosomal abnormality detected both in Ewing's sarcoma and in primitive neuroectodermal tumor cells. The translocation results in an EWS-Fli1 fusion gene, made up of the $5^{\prime}$ half of the $E W S$ gene on chromosome 22 fused to the $3^{\prime}$ half of the Fli1 gene on chromosome 11. Recent studies have evaluated possible roles of the fusion gene products. However, the biological significance of EWS-Fli1 is still unknown. Using a competitive polymerase chain reaction technique, we show here that there might be a correlation between the expression levels of the EWS-Fli1 fusion gene and the proliferative activities of Ewing's sarcoma and primitive neuroectodermal tumor cells. When the EWS-Fli1 expression is inhibited by antisense oligodeoxynucleotides against the fusion RNA, the growth of the tumor cells is significantly reduced both in vitro and in vivo. The data further indicate the growth inhibition of the cells by the antisense sequence might be mediated by $G_{0} / G_{1}$ block in the cell cycle progression. These results suggest that EWS-Fli1 may play an important role in the proliferation of the tumor cells, and the EWS-Fli1 fusion RNA could be used as a target to inhibit the growth of Ewing's sarcoma and primitive neuroectodermal tumor with the specific antisense oligonucleotide. (J. Clin. Invest. 1997. 99:239-247.) Key words: small round cell tumors • chromosomal translocation $\bullet$ competitive polymerase chain reaction • gene therapy $\bullet$ cell cycle
\end{abstract}

\section{Introduction}

Specific chromosomal translocations are often associated with particular types of hematopoietic and solid tumors $(1,2)$. Investigation of solid tumor translocations has concentrated on sarcomas, where the cytogenetics have been well studied. Four of these translocations have been identified and found to result in fusion proteins in Ewing's sarcoma (ES) ${ }^{1}$ and primitive neu-

Address correspondence to Kazuhiro Tanaka, Laboratory of Developmental Biology, National Institute of Dental Research, NIH, Building 30, Room 411, 30 Convent DR MSC 4370, Bethesda, MD 208924370. Phone: 301-496-1108; FAX: 301-402-0897; E-mail: ktanaka@yoda. nidr.nih.gov

Received for publication 16 April 1996 and accepted in revised form 12 November 1996.

1. Abbreviations used in this paper: C-PCR, competitive PCR; ES, Ewing's sarcoma; PNET, primitive neuroectodermal tumor; RTPCR, reverse transcriptase PCR.

J. Clin. Invest.

(C) The American Society for Clinical Investigation, Inc.

0021-9738/97/01/239/09 \$2.00

Volume 99, Number 2, January 1997, 239-247 roectodermal tumor (PNET) (3), liposarcoma $(4,5)$, rhabdomyosarcoma $(6,7)$, and synovial sarcoma (8).

The translocation $\mathrm{t}(11 ; 12)(\mathrm{q} 24 ; \mathrm{q} 12)$ is a karyotypic abnormality detected in $\sim 90 \%$ of both ES and PNET (9-11). As a result of this translocation, two genes, the EWS gene on chromosome 22 and the Fli1 gene on chromosome 11, are fused in ES and PNET cells (3). The Flil gene is a member of the ETS family of transcription factors and contains a sequence-specific DNA-binding domain (12). One portion of the EWS gene appears to encode a RNA-binding domain (3), but its function is not known. The translocation $\mathrm{t}(11 ; 12)(\mathrm{q} 24 ; \mathrm{q} 12)$ results in the EWS promoter-driven expression of a fusion transcript that produces an EWS-Fli1 chimeric protein containing EWSderived sequences at its amino terminus fused to the carboxyterminal region of Fli1. Recent studies have suggested possible roles of the EWS-Fli1 protein in the tumorigenicity of ES and PNET. The EWS-Fli1 fusion gene constructs transfected into murine fibroblasts might act as a transforming gene (13). Some groups have reported that the EWS-Fli1 chimeric protein may function as an aberrant transcription factor in ES and PNET cells (14-17), and some of the target genes modulated by the EWS-Fli1 protein have been identified (18). Recent studies have shown that stable expression of antisense EWS-Fli1 in ES cells by transfecting the antisense EWS-Fli1 expression plasmid resulted in growth reduciton of the ES cells, suggesting possible roles of the fusion products in tumorigenesis $(19,20)$. However, the mechanisms of oncogenesis by the chimeric protein are still not known.

Antisense oligodeoxynucleotides are able to enter all cells relatively easily and have the potential to target specific genes $(21,22)$. Antisense DNA oligomers are short sequences complementary to a site on the RNA transcript of a target gene. Hybridization of the antisense oligomer and the target RNA by affinity is in many ways superior to that of antibody-ligand binding. Antisense DNA can inhibit the expression of the protein product of the transcript through several mechanisms, including blocking ribosomal translation of the RNA transcript (23), triggering RNase $\mathrm{H}$ degradation of the target RNA (24), and interfering with the processing of pre-mRNA (25). The efficacy of antisense DNA inhibition has been demonstrated in cell culture against a variety of genes $(21,22)$. Towards understanding biological consequences of EWS-Fli1, we examined the effects of antisense DNA complementary to the EWS-Fli1 chimeric RNA on the phenotype of both ES and PNET cells. Our data demonstrate that there is a correlation between the expression levels of the EWS-Fli1 fusion gene and the proliferative activities of both ES and PNET cells. When EWS-Fli1 expression was inhibited using the antisense sequence, a significant reduction in cell proliferation was achieved both in vitro and in vivo. The data further indicated that the growth inhibition of ES and PNET cells by antisense oligonucleotide was mediated by $G_{0} / G_{1}$ block in the cell cycle progression. These results suggest that EWS-Fli1 may be responsible for continu- 
ous proliferation of ES and PNET cells, and that inhibition of EWS-Fli1 by the antisense oligonucleotide might be an effective way of controlling the growth of these tumors.

\section{Methods}

Cell lines. Human ES cell lines, SK-ES-1 and RD-ES, human PNET cell line SK-N-MC, human fibrosarcoma cell line HT1080, human neuroblastoma cell line IMR-32, and human fibroblast cells, MRC-5 and IMR-90, were obtained from the American Type Culture Collection (Rockville, MD). Human PNET cell line PNKT-1 was established and characterized in our laboratory (26). These cells were maintained in DME supplemented with $10 \%$ FBS, $100 \mu \mathrm{g} / \mathrm{ml}$ penicillin, and $100 \mu \mathrm{g} / \mathrm{ml}$ streptomycin and incubated at $37^{\circ} \mathrm{C}$ in a humidified atmosphere containing $5 \% \mathrm{CO}_{2}$ in air.

For the determination of cell population doubling time, the cells were seeded $10^{5}$ viable cells/dish with $5 \mathrm{ml}$ of medium in triplicate 60-mm culture dishes (Falcon Labware, NJ). The number of viable cells in each dish was counted every $24 \mathrm{~h}$ for $5 \mathrm{~d}$ by the trypan blue exclusion test. The numbers of viable cells treated with antisense oligonucleotide were adjusted to equal those of untreated or sense oligonucleotide-treated cells at times of harvest for RNA and Western analyses.

Animals. 6-wk-old female athymic Balb/c nu/nu mice were obtained from Japan SLC Co., Ltd. (Hamamatsu, Japan). Throughout the experiments, mice were maintained in a laminar flow cabinet under specific pathogen-free conditions receiving standard feed and water ad libitum. All experiments on animals were conducted in accordance with the guidelines of the Animal Center of Kyushu University.

Oligodeoxynucleotides. Antisense and sense phosphorothioate oligodeoxynucleotides purified by high performance liquid chromatography were purchased from Kurabo Biomedicals (Osaka, Japan). Five antisense oligomers directed against the breakpoint or sequences including the ATG initiation codon of the EWS-Fli1 fusion gene were synthesized. The sequences of the antisense oligonucleotides were: ESAS1, CTGTAATCCGTGGACGCCATTTTCT (nucleotides 39-63; reference 3); ESAS2, ATCCGTGGACGCCATTTTCTCTCCT (nucleotides 34-58); ESAS3, TGGACGCCATTTTCTCTCCTTCCTC (nucleotides 29-53); BPAS1, GACTGAGTCATAAGAAGGGTTCTGC (nucleotides 832-856 of type 1); and BPAS2, CATAAGAAGGGTTCTGCTGCCCGTA (nucleotides $824-848$ of type 1). The corresponding sense sequences were used as controls. A scrambled oligonucleotide (ESSC2, GTTATCTATTCTCCGACCGTCGTCC) corresponding to antisense ESAS2 was also synthesized and used as a control. Oligonucleotide primers for reverse transcription PCR (RT-PCR) were also synthesized by Kurabo Biomedicals. The primers used were: CCACTAGTTACCCACCCCAAACTG and GTGATACAGCTGGCGTTGGCG for EWS-Fli1 cDNA amplification; CCACTAGTTACCCACCCCAAACTG and CCTCCTCTATCTCCTCCACGGC for EWS; and TTACCAAAAGTGGCCCACTA and GAAAGATGGTGAACTATGCC for ribosomal cDNA. Each cDNA carried the following sequences: from 753 to 1150 base positions for EWS-Fli1 (type 2); from 753 to 1530 base positions for EWS (3); and from 1501 to 1846 base positions for ribosomal cDNA (27).

$R T-P C R$. Total cellular RNA was extracted from each cell line as described (28). The RNA preparations were evaluated for concentration by $\mathrm{OD}_{260}$ absorption and for quality by agarose gel electrophoresis followed by ethidium bromide staining. $2 \mu \mathrm{g}$ of total RNA were reverse-transcribed with an oligo-d $(\mathrm{T})_{18}$ primer using a T-primed first-strand kit (Pharmacia LKB Biotechnology, Piscataway, NJ) according to the manufacturer's instructions. Final first-strand cDNA reaction was stored at $-80^{\circ} \mathrm{C}$ until use for PCR. PCR reactions for each set of primers were performed separately in a total volume of 50 $\mu l$ containing $10 \mathrm{mM}$ Tris- $\mathrm{HCl}, \mathrm{pH} 8.3,50 \mathrm{mM} \mathrm{KCl}, 1.5 \mathrm{mM} \mathrm{MgCl}_{2}$, $0.1 \mathrm{mM}$ of each dNTP, $0.4 \mu \mathrm{M}$ of each primer, $2.5 \mathrm{U}$ of Taq DNA polymerase, and $2 \mu \mathrm{l}$ of the reverse transcription reaction. The amplification profile consisted of denaturation at $94^{\circ} \mathrm{C}$ for $1 \mathrm{~min}$, primer annealing at $66^{\circ} \mathrm{C}$ for $1 \mathrm{~min}$, and extension at $72^{\circ} \mathrm{C}$ for $1 \mathrm{~min}$, for a total of 35 cycles on a PCR thermal cycler (DNA Thermal Cycler 480; Perkin-Elmer Corp., Norwalk, CT). To confirm that an equal amount of RNA was reverse-transcribed to cDNA, PCR amplification of the $r R N A$ gene was performed using the primers described above. Reaction products were separated on a $1.2 \%$ agarose gel and visualized by ethidium bromide staining. Data were analyzed using a National Institutes of Health (NIH) image Ver 1.56.

Competitive PCR assay. The 398-bp PCR fragment of EWS-Fli1 type 2 cDNA and the 778-bp fragment of EWS were subcloned into pBSKS $(-)$ (Stratagene Inc., La Jolla, CA). Each cDNA was sequenced and confirmed to possess a sequence identical to the published one (3). The EWS-Fli1 cDNA clone was digested in the middle of the sequence by EcoRI and BamHI, and ligated with the EcoRIBamHI fragment of MMP9 cDNA (nucleotides 1204-1950; reference 29). The EWS cDNA clone was also digested in the middle of the sequence by BamHI and XbaI, and ligated with the BamHI-XbaI fragment of MMP9 cDNA (nucleotides 390-1274; reference 29). The mutant EWS-Fli1 and EWS cDNA constructs, which produced a 973- and a 1,276-bp fragment, respectively, were amplified by the same primers used for EWS-Fli1 and EWS cDNA amplification, and were used as competitive templates. Serial dilutions of the competitive templates were added to PCR tubes containing all the amplification reagents plus either EWS-Fli1 or EWS cDNA template. The reaction mixtures were then amplified, and the products were separated on agarose gel and analyzed as described above. The mutant/test cDNA ratio for each reaction tube was plotted as a function of the amount of mutant template and a straight line was drawn by linear regression analysis. The quantity of cDNA in the test sample was the amount at which the mutant/test band density ratio was equal to one.

Monitoring of first-strand synthesis. To test the yield and the efficiency of the reverse transcriptase reaction, $2 \mu \mathrm{g}$ of total RNA was subjected to reverse transcription as described above, but with $2 \mu \mathrm{l}$ of $\left[\alpha-{ }^{32} \mathrm{P}\right] \mathrm{dCTP}(10 \mathrm{mCi} / \mathrm{ml}$, Amersham Corp., Arlington Heights, IL) added to the reaction. The total volume of the RT reaction was $35 \mu l$. $1 \mu \mathrm{l}$ of the reaction was removed for the determination of total counts. After the RT reaction, the labeled cDNA was purified by chromatography on a spun column of Sephadex G-50 (Pharmacia LKB Biotechnology) to remove any unincorporated radioactive nucleotides. The radioactivity of the labeled cDNA was measured with a liquid scintillation counter. The amount of synthesized cDNA was calculated as described (30).

Western blot analysis. Western blotting was carried out as described (28) with several modifications. Cells were incubated with or without $10 \mu \mathrm{M}$ oligonucleotides in serum-free medium for $48 \mathrm{~h}$. After incubation, the cells were washed twice in ice-cold PBS, and lysed in $10 \mathrm{mM}$ Tris-Cl, pH 7.4, $100 \mathrm{mM} \mathrm{NaCl}, 1 \mathrm{mM}$ EDTA, $1 \%$ Triton X, $0.5 \%$ deoxycholate, $0.1 \%$ SDS, $100 \mu \mathrm{g} / \mathrm{ml}$ phenylmethylsulfonyl fluoride, $10 \mu \mathrm{g} / \mathrm{ml}$ leupeptin, and then centrifuged at 15,000 rpm for 30 min. $70 \mu \mathrm{g}$ lysate protein was electrophoresed on $10 \%$ polyacrylamide gels and the proteins were then electrotransferred onto nitrocellulose membranes. The membranes were blocked in 5\% BSA, $0.05 \%$ Tween 20 in buffered saline $(0.9 \% \mathrm{NaCl}, 10 \mathrm{mM}$ Tris-Cl, $\mathrm{pH} 7.4)$ for $1 \mathrm{~h}$ at $52^{\circ} \mathrm{C}$, and then reacted with the antibody against the $\mathrm{COOH}$-terminal portion of murine Fli1 (Santa Cruz Biotechnology, Santa Cruz, CA) for $12 \mathrm{~h}$ at $4^{\circ} \mathrm{C}$. The blots were rinsed in buffered saline containing $0.05 \%$ Tween 20 and reacted with horseradish peroxidase-conjugated secondary antibody for $1 \mathrm{~h}$ at room temperature. Visualization was achieved by chemiluminescence (Amersham Corp.) followed by $\mathrm{x}$-ray film exposure. Data were analyzed using an NIH image Ver 1.56.

Although the Fli1 antibody was raised against the $\mathrm{COOH}$-terminal 19 amino acids of murine Fli1, it cross-reacts well with human Fli1. The Fli1-specific antibody recognizes the EWS-Fli1 fusion protein at its $\mathrm{COOH}$-terminal portion derived from Fli1, as previously described (16).

Cell growth assay. Various cell lines were seeded on 35-mm cul- 
ture dishes (Falcon Labware) at $10^{5}$ viable cells/dish with $1.5 \mathrm{ml}$ of medium, and incubated at $37^{\circ} \mathrm{C}$ in a humidified atmosphere containing $5 \% \mathrm{CO}_{2}$ in air. $24 \mathrm{~h}$ later, the media were replaced with serumfree media containing various concentrations $(0-30 \mu \mathrm{M})$ of antisense or sense oligonucleotides, and the media supplemented with the oligomers were replaced every $24 \mathrm{~h}$. After various time intervals $(0-120 \mathrm{~h})$, cell numbers were determined using a hemocytometer. Since the cell viability determined by the trypan blue exclusion test was more than $95 \%$ throughout the experiments, the concentrations of oligonucleotides were not toxic. All experiments were carried out in triplicate and repeated twice.

Oligonucleotide administration to nude mice. SK-N-MC cells were harvested from $60 \%$ confluent monolayer cultures, resuspended with PBS at $2 \times 10^{7}$ viable cells $/ \mathrm{ml}$, and subcutaneously inoculated into athymic mice $\left(5 \times 10^{6}\right.$ viable cells/mouse $) .7 \mathrm{~d}$ after tumor inoculation, when the subcutaneous tumor had grown to a visible size, $0.1 \mathrm{ml}$ of PBS containing $1 \mathrm{mM}$ antisense or sense oligonucleotides was injected into the established tumor (day 1). Four additional injections were given at days 3,5,7, and 9. The tumor volume was estimated during the experiments by two perpendicular measurements, length (a) and width (b), of the tumor and was calculated $a a^{2} / 2$. The animals were killed $28 \mathrm{~d}$ after the first oligomer inoculation, and the subcutaneous tumors were excised and weighed.

Flow cytometric analysis and cell cycle distribution. SK-N-MC cells were incubated with or without oligonucleotides $(10 \mu \mathrm{M})$ for 24,48 , or $72 \mathrm{~h}$, and prepared for flow cytometric analysis after staining of the DNA with propidium iodide as described previously (31). Fluorescence intensity in the cell nucleus was measured with a FACScan ${ }^{\circledR}$ (Becton Dickinson \& Co., San Jose, CA). For each sample, 10,000 cells were analyzed, and the results were processed by the computer program CellFit (Becton Dickinson \& Co.)

Statistical analysis. All statistical analyses were carried out according to the Mann-Whitney U test.

\section{Results}

Identification of EWS-Fli1 fusion gene expression in ES and PNET cells. The expression of the EWS-Fli1 fusion gene in ES cell lines, SK-ES-1 and RD-ES, and PNET cell lines, SK-NMC and PNKT-1, was determined by RT-PCR. Amplification of cDNAs from SK-ES-1 and RD-ES resulted in 398-bp products, whereas those from SK-N-MC and PNKT-1 resulted in 332-bp products (data not shown). Nucleotide sequence analysis of these products revealed that the 398- and 332-bp fragments were identical to EWS-Flil type 2 and type 1 fusion genes, respectively (data not shown) (32). HT1080 fibrosarcoma, MRC-5 fibroblast, and IMR-32 neuroblastoma cells did not show any fusion gene products (data not shown).

Competitive PCR quantification procedure of EWS-Fli1 $m R N A$. For quantification of EWS-Fli1 mRNA, a competitive PCR (C-PCR) method was established by developing a mutated template of EWS-Fli1 cDNA, which was coamplified with endogenous EWS-Fli1 cDNA in the same tube as the internal standard. Accordingly, mutant cDNA gave an amplification product of $973 \mathrm{bp}$ in size, compared with 332 or $398 \mathrm{bp}$ for endogenous products. The use of the same primer-binding sites resulted in identical amplification efficiency. When coamplified in the same vial, both mutant and endogenous EWSFli1 cDNA competed for primer bindings in a concentrationdependent manner. As shown in Fig. 1, increasing amounts of mutant EWS-Fli1 cDNA progressively inhibited amplification of endogenous EWS-Fli1 cDNA.

To enhance accuracy, a standard curve for the quantification of the endogenous EWS-Fli1 cDNA was established by serial dilutions of the original mixture. The decreasing intensi- ties of the mutant and endogenous PCR products reflected the rising degree of dilution, thereby minimizing sample-to-sample variations between individual PCR vials (data not shown). To evaluate the efficiency of the reverse transcriptase reaction, the amount of synthesized cDNA from total RNA was calculated. Results indicated that RT reaction efficiency under the condition used was $60.4 \pm 5.9 \%(n=6)$. This value was used as a correction factor for the calculation of EWS-Fli1 mRNA concentrations in the samples. Increasing reverse transcription times or reverse transcriptase concentrations did not affect the ratio of endogenous to mutant EWS-Fli1, indicating stable cDNA levels after reverse transcription procedures (data not shown).

To ensure that quantification occurs within the exponential phase of PCR, amplification products of the same reaction mixture were amplified for up to 40 cycles and aliquots were taken out at different time points during the amplification (from 30 to 40 cycles). Results indicated that the amplification

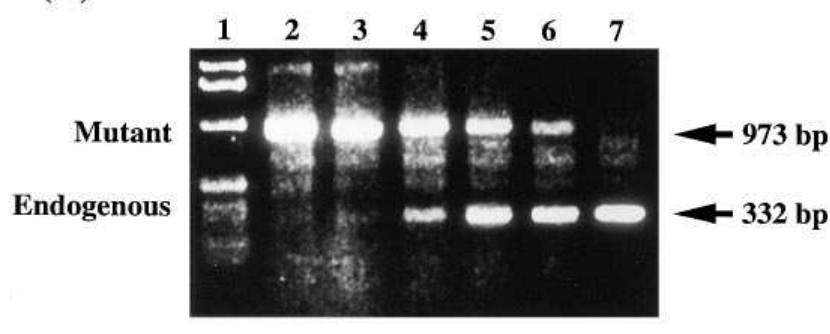

rRNA

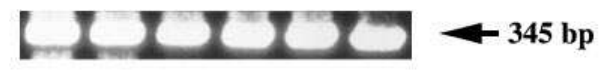

(B)

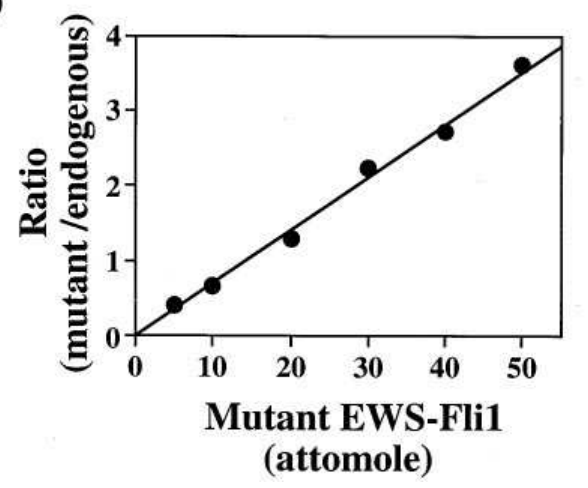

Figure 1. C-PCR quantification of EWS-Fli1 cDNA. A constant amount of the RT product and a decreasing amount of the mutant EWS-Fli1 cDNA were added to a series of tubes containing all the PCR reagents. The mutant cDNA differed from the endogenous EWS-Fli1 cDNA due to a 575-bp insertion in the middle of the type 2 fusion molecule. Increasing amounts of the mutant cDNA progressively inhibited coamplification of the tested EWS-Fli1 cDNA. (A) A representative ethidium bromide-stained gel after PCR amplification of RT products from SK-N-MC. The rRNA products are shown as quality control of the RNAs. Lane 1, molecular weight marker; lanes 2-7 contain mutant EWS-Fli1 template at 50, 40, 30, 20, 10, and 5 amol, respectively. $(B)$ Ratio of mutant/endogenous EWS-Fli1 densitometric values obtained from the gel in $A$ vs. the amount of mutant cDNA added to each tube. The amount of cDNA in the test sample was calculated from the equivalence point at which the ratio was equal to one. The correlation coefficient $=0.993$. 


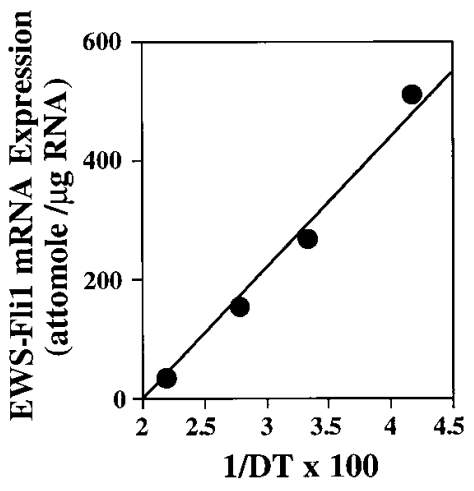

Figure 2. Relationship between EWS-Fli1 mRNA expression and cell growth. The fusion gene expression levels in ES and PNET cells were calculated from the C-PCR data. To determine the cell population doubling time, the cells were seeded in triplicate 60-mm culture dishes at a density of $10^{5}$ viable cells/ dish, and the number of viable cells was counted every $24 \mathrm{~h}$ for $5 \mathrm{~d}$. The cell growth was represented by a reciprocal of the cell population doubling time $(D T)$ of each cell line. The data represent the means of the three experiments. The correlation coefficient $=0.991$.

was linear at 35 cycles, at which point quantitative analysis was carried out (data not shown).

Relationship between expression levels of EWS-Fli1 mRNA and cell proliferation. The EWS-Fli1 mRNA expression levels in both ES and PNET cell lines were calculated from the C-PCR data. The values of EWS-Fli1 mRNA were 508.2 \pm 25.2 $\mathrm{amol} / \mu \mathrm{g}$ total RNA for SK-N-MC, $268.2 \pm 18.6 \mathrm{amol} / \mu \mathrm{g}$ total RNA for PNKT-1, 153.3 $\pm 22.1 \mathrm{amol} / \mu \mathrm{g}$ total RNA for RD-ES, and $35.8 \pm 10.3 \mathrm{amol} / \mu \mathrm{g}$ total RNA for SK-ES-1. On the other hand, cell population doubling times of SK-N-MC, PNKT-1, RD-ES, and SK-ES-1 were 24.0, 30.2, 36.0, and $45.6 \mathrm{~h}$, respectively. As shown in Fig. 2, the expression level of EWS-Fli1 mRNA was in proportion to a reciprocal of the doubling time $(r=0.991)$, suggesting that the ES and PNET cells that express a higher level of EWS-Fli1 mRNA might exhibit rapid cell growth.

Inhibition of proliferation of ES and PNET cells by antisense oligonucleotide. The effects of EWS-Fli1 antisense oligonucleotides on the growth of ES and PNET cells were examined next. Five antisense phosphorothioate oligonucleotides directed against the breakpoint or the sequences including ATG initiation codon of the EWS-Fli1 fusion gene were syn-

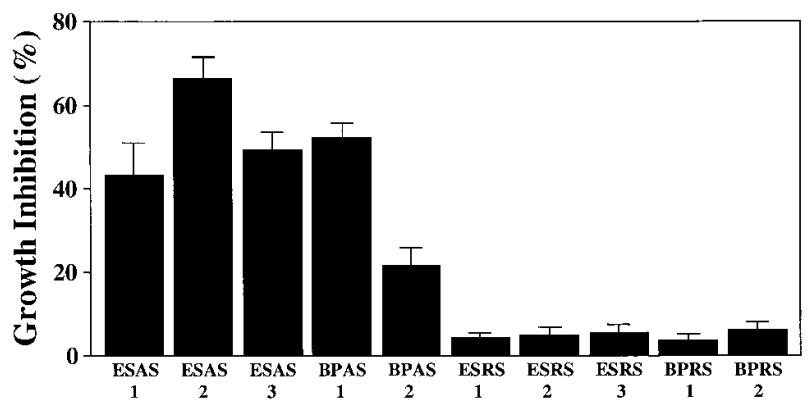

Figure 3. Effects of antisense and sense oligonucleotides on SK-N-MC cell proliferation. The cells $\left(10^{5}\right.$ viable cells/dish $)$ were incubated with or without $10 \mu \mathrm{M}$ of oligonucleotides summarized in Table I. The medium containing each oligomer was replaced every $24 \mathrm{~h}$. After a 72-h incubation, the number of viable cells was counted. The data represent the means of the six experiments. The bars represent SEM.

thesized. The sense oligonucleotides corresponding to the antisense sequences were also synthesized and used as controls (Table I). As shown in Fig. 3, the antisense oligonucleotides inhibited the growth of SK-N-MC cells, while sense oligonucleotides produced no effect. The ability of the antisense oligomers to inhibit the proliferation of SK-N-MC cells varied widely. BPAS2 was of minimal efficacy, showing only $21.6 \pm 4.1 \%$ inhibition $(P<0.05)$, while BPAS1 was very effective at $52.0 \pm 3.6 \%$ inhibition $(P<0.01)$. ESAS2 was the most effective at $66.4 \pm 5.2 \%$ inhibition $(P<0.01)$, and was mainly used in the subsequent experiments.

ESAS2 was also effective on various ES and PNET cells. The oligonucleotide prolonged the population doubling times of SK-N-MC, PNKT-1, RD-ES, and SK-ES-1 cells to 46.1, $64.3,73.2$, and $81.6 \mathrm{~h}$, respectively $(P<0.01)$, while sense or scrambled oligonucleotide had no effect (Fig. 4). This effect was dose dependent over the range of 1-30 $\mu \mathrm{M}$ (Fig. 5). When the cells were cultured with $10 \mu \mathrm{M}$ ESAS2 for $72 \mathrm{~h}$, the number of SK-N-MC, PNKT-1, RD-ES, and SK-ES-1 cells were $39.4,45.9,52.3$, and $62.3 \%$ of the control, respectively $(P<0.01)$. The cell viability, determined by the trypan blue exclusion test, was not significantly affected by these treatments (data not

Table I. Summary of Synthesized Antisense and Sense Phosphorothioate Oligonucleotide Sequences to EWS-Fli1 cDNA

\begin{tabular}{lll}
\hline Oligonucleotide & \multicolumn{1}{c}{ Sequence $\left(5^{\prime} \rightarrow 3^{\prime}\right)$} & cDNA target \\
\hline ESAS1 & CTGTAATCCGTGGACGCCATTTTCT & Nucleotides 39-63 \\
ESAS2 & ATCCGTGGACGCCATTTCTCTCCT & Nucleotides 34-58 \\
ESAS3 & TGGACGCCATTTTCTCTCCTTCCTC & Nucleotides 29-53 \\
BPAS1 & GACTGAGTCATAAGAAGGGTTCTGC & Nucleotides 832-856 \\
BPAS2 & CATAAGAAGGGTTCTGCTGCCCGTA & Nucleotides 824-848 \\
ESRS1 & AGAAAATGGCGTCCACGGATTACAG & Sense for ESAS1 \\
ESRS2 & AGGAGAGAAAATGGCGTCCACGGAT & Sense for ESAS2 \\
ESRS3 & GAGGAAGGAGAGAAAATGGCGTCCA & Sense for ESAS3 \\
BPRS1 & GCAGAACCCTTCTTATGACTCAGTC & Sense for BPAS1 \\
BPRS2 & TACGGGCAGCAGAACCCTTCTTATG & Sense for BPAS2 \\
ESSC2 & GTTATCTATTCTCCGACCGTCGTCC & Scrambled for ESAS2
\end{tabular}

Five antisense phosphorothioate oligonucleotides 25 bases in length targeted to the breakpoint or the sequences including the ATG initiation codon of the EWS-Fli1 fusion gene (ESAS1, 2, and 3, and BPAS1 and 2) were synthesized. Sense oligonucleotides corresponding to antisense and a scrambled oligonucleotide corresponding to antisense ESAS2 were also synthesized. Sequences are shown $5^{\prime}$ to $3^{\prime}$, and the nucleotide location on EWS-Fli1 cDNA (3) is shown. 


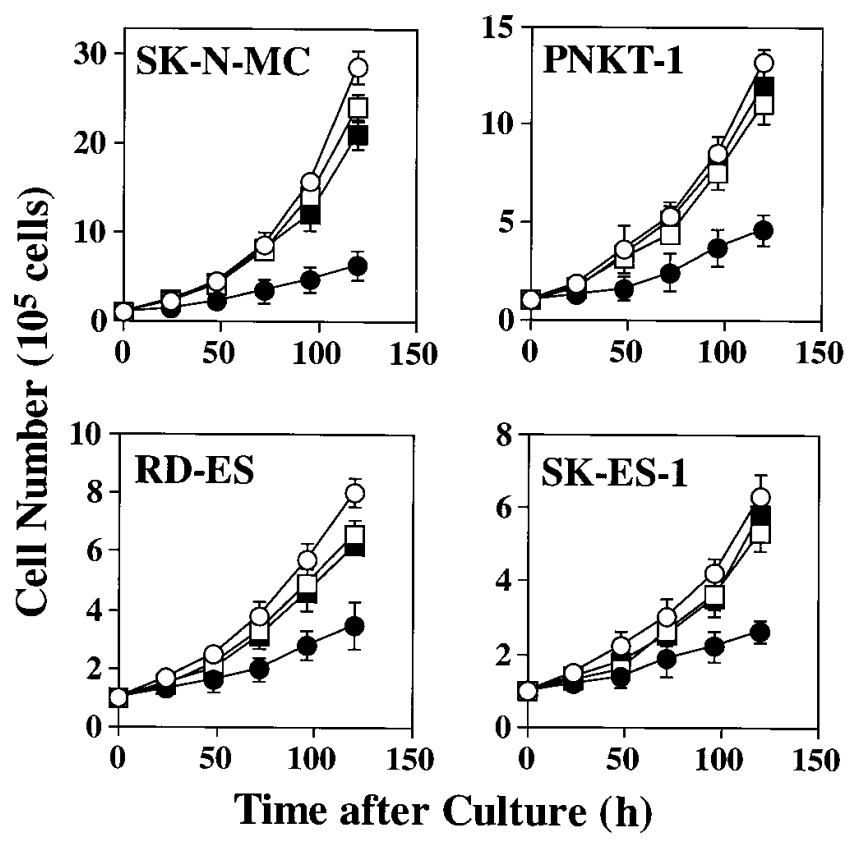

Figure 4. Effects of antisense oligonucleotide on growth of SK-N-MC, PNKT-1, RD-ES, and SK-ES-1. The cells were incubated with or without $10 \mu \mathrm{M}$ of ESAS2, ESRS2, or ESSC2 oligonucleotides. After various time intervals $(0-120 \mathrm{~h})$, the cell number was counted as described in the legend to Fig. 3. The data represent means of the six experiments. The bars represent SEM. $\bigcirc$, control; -, ESAS2 antisense oligomer; $\square$, ESRS2 sense oligomer; $\mathbf{\square}$, ESSC2 scrambled oligomer.

shown). None of the oligonucleotides affected the growth of HT1080, IMR-32, MRC-5, or IMR-90 cells (Fig. 5). The results suggested that the product of the EWS-Flil transcript might be responsible for continuous growth of the ES and PNET cells.

Effects of antisense oligomer on expression of EWS-Fli1 and EWS genes. To investigate whether the antisense oligonucleotide had a specific effect on target mRNA, C-PCR was performed to quantify EWS-Fli1 fusion mRNA. In the presence of $10 \mu \mathrm{M}$ ESAS2, the EWS-Fli1 mRNA level in SK-N-MC was $12.7 \pm 2.1 \mathrm{amol} / \mu \mathrm{g}$ total RNA, whereas in the untreated line the level was $521 \pm 16.4 \mathrm{amol} / \mu \mathrm{g}$ total RNA. The sense and
(A)

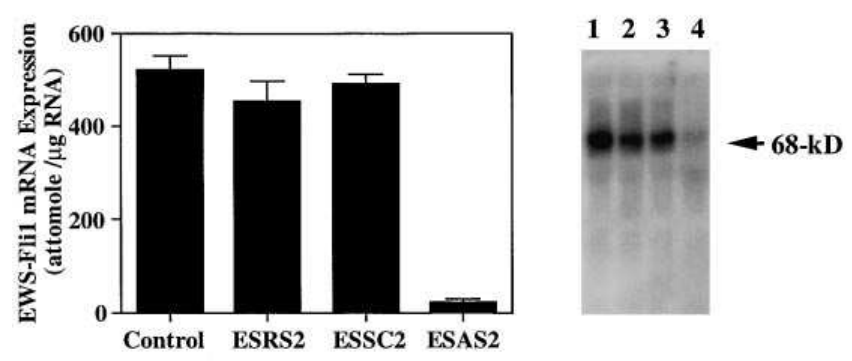

(C)

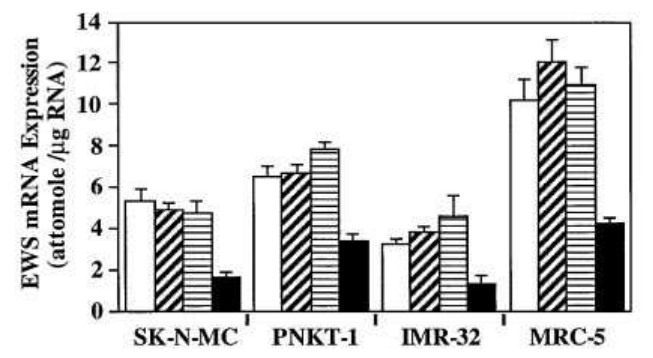

Figure 6. Effects of antisense oligonucleotide on EWS-Fli1 and EWS expression. (A) EWS-Fli1 gene expression modulated by antisense oligomer. SK-N-MC cells were incubated with or without $10 \mu \mathrm{M}$ ESAS2, ESRS2, or ESSC2 for $48 \mathrm{~h}$, and then total RNA was extracted and subjected to C-PCR analysis as described in the legend to Fig. 1. The expression levels of EWS-Fli1 mRNA in the cells were calculated from the C-PCR data. The value represent means of the three experiments. The bars represent SEM. $(B)$ Western blot analysis of EWS-Fli1 proteins. SK-N-MC cells were incubated with $10 \mu \mathrm{M}$ of ESAS2, ESRS2, or ESSC2 for $48 \mathrm{~h}$, and the cells were harvested and subjected to Western blotting with anti-Fli1 antibody. The $68-\mathrm{kD}$ band for EWS-Fli1 protein was detected. Lane 1, control; lane 2, ESRS2; lane 3, ESSC2; lane 4, ESAS2. (C) Normal EWS gene expression modulated by antisense oligonucleotide. Various cell lines were incubated in the presence or absence of $10 \mu \mathrm{M}$ ESAS2, ESRS2, or ESSC2 for $48 \mathrm{~h}$, and total RNA was extracted and prepared for C-PCR analysis. The expression levels of EWS mRNA in the cells were calculated from the C-PCR data. The data represent the means of the three experiments. The bars represent SEM. $\square$, Control; $\square$, ESRS2; 目, ESSC2; $\mathbf{a}, \mathrm{ESAS} 2$.

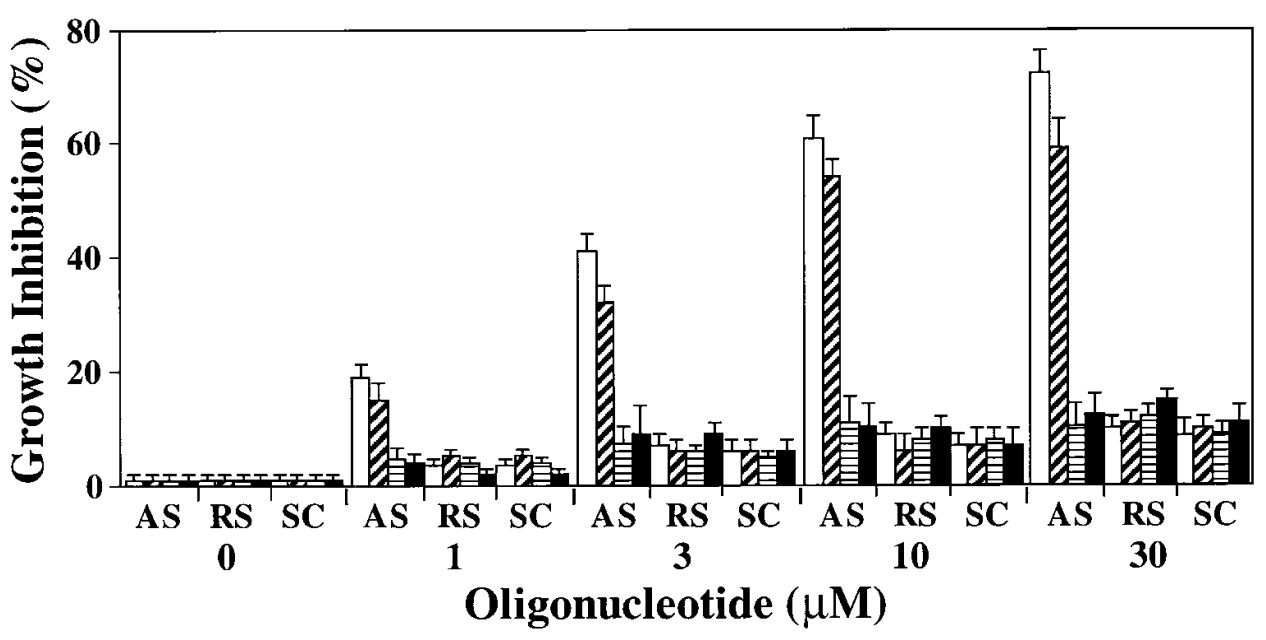

Figure 5. Effects of different doses of antisense oligonucleotide on growth of SK-N-MC, PNKT-1, IMR-32, and MRC-5. The cells were cultured with various concentrations of ESAS2 $(A S)$, ESRS2 $(R S)$, or ESSC2 (SC) oligonucleotides for $72 \mathrm{~h}$, and the cell number was counted as described in the legend to Fig. 3. The data represent means of the six experiments. The bars represent SEM. $\square$, SK-N-MC; $\square$, PNKT-1; 目, IMR-32; MRC-5. 


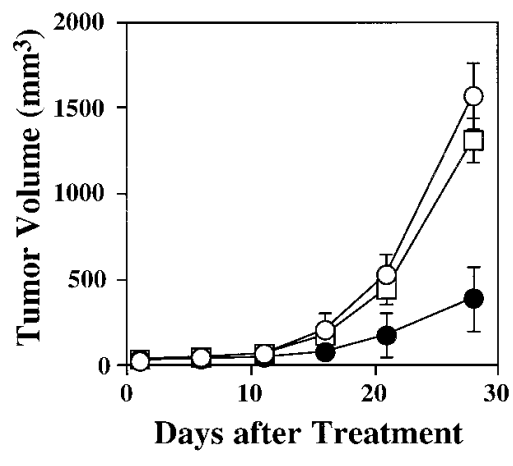

tions were given at days 3, 5, 7, and 9. The tumor volume was measured during the experiments. The data represent the means of six samples. The bars represent SEM. $\bigcirc$, control; $\bullet$ ESAS2; $\square$, ESRS2.

scrambled oligomers produced no significant effect on the level of mRNA. There was an $\sim 40$-fold decrease in EWS-Fli1 mRNA detected in the SK-N-MC line $(P<0.001$; Fig. $6 A)$. When the cells were incubated with $10 \mu \mathrm{M}$ BPAS1 for $48 \mathrm{~h}$, the EWS-Fli1 mRNA level of the treated cells calculated from C-PCR data was $\sim 28$-fold lower than that of control cells (data not shown).

To further prove that the decrease in EWS-Fli1 mRNA levels in the antisense-treated cells also resulted in a comparable reduction in the protein, EWS-Fli1 protein levels were determined by Western blot analysis. The amount of EWS-Fli1 protein from cells treated with $10 \mu \mathrm{M}$ ESAS2 for $48 \mathrm{~h}$ was reduced to $\sim 10 \%$ of the levels seen in control, ESRS2- and ESSC2-treated cells (Fig. $6 \mathrm{~B}$ ). Similar results were obtained in the other ES and PNET cells (data not shown).

Since ESAS2 was targeted against the 5' end of EWS-Fli1, including the AUG initiation codon, the antisense oligomer might bind to normal EWS mRNA. Therefore, we also assessed the changes of the EWS mRNA levels induced by the antisense oligomer using the C-PCR assay as described above. The intrinsic $E W S$ mRNA was detected as a 778-bp PCR product, not only in ES and PNET cell lines, but also in IMR-32 and MRC-5. However, the expression levels of EWS genes were very low in comparison with those of $E W S$-Flil. There was no correlation between the EWS expression levels in the cells and the growth rate of the cells. When these cells were in-

Table II. Weight of Subcutaneous Tumors of Nude Mice Inoculated with $S K-N-M C$ Cells and Treated with ESAS2 Antisense or ESRS2 Sense Oligonucleotides

\begin{tabular}{|c|c|c|}
\hline Treatment & Tumor weight & $\begin{array}{c}\text { Average } \\
\text { tumor weight }\end{array}$ \\
\hline & $m g$ & $m g$ \\
\hline $\begin{array}{l}\text { Control } \\
\qquad(n=6)\end{array}$ & 1,673.4, 1.436.2, 1,209.7, 1,158.0, 1,092.5, 932.1 & $1,250.3$ \\
\hline $\begin{array}{l}\text { ESRS2 } \\
\quad(n=6)\end{array}$ & $1,418.5,1,296.9,1,270.0,1,064.4,1,062.3,912.7$ & $1,170.6$ \\
\hline $\begin{array}{l}\text { ESAS2 } \\
\quad(n=6)\end{array}$ & $451.9,417.3,379.0,303.8,226.2,57.4$ & 305.9 \\
\hline
\end{tabular}

$7 \mathrm{~d}$ after the cell inoculation, injections of oligonucleotides were started. Mice received a total dose of $500 \mathrm{nmol}$ of each oligonucleotide in five injections. The animals were killed $28 \mathrm{~d}$ after the first oligonucleotide injection, and the subcutaneous tumors were excised and weighed.
Figure 8. Flow cytometric analysis of SK$\mathrm{N}-\mathrm{MC}$ cells exposed to antisense oligonucle-

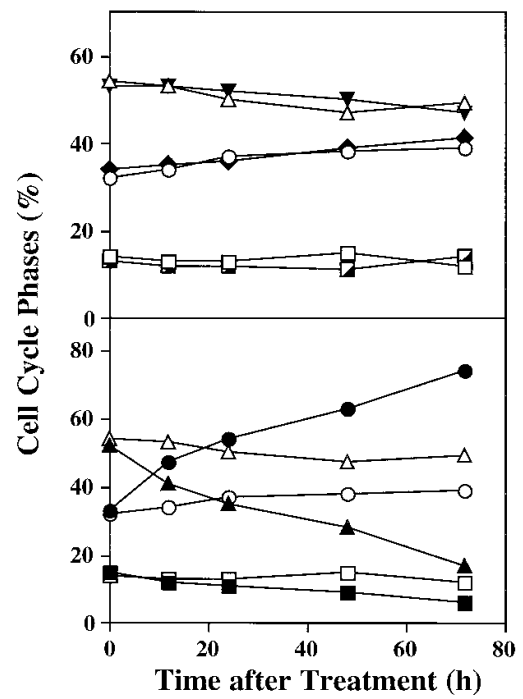

otide. The SK-N-MC cells were incubated or ESAS2 $(10 \mu \mathrm{M}$ each) for different time intervals $(0,24,48$, or $72 \mathrm{~h}$ ). Then the cells were processed for propidium iodide staining and FACS ${ }^{\circledR}$ analysis. The percentage of cells in each cell cycle phase is shown. $\bigcirc$, control/ $\mathrm{G}_{0}-\mathrm{G}_{1} ; \triangle$, control/S; $\square$, control $/ \mathrm{G}_{2}+\mathrm{M}$; ESRS $2 / \mathrm{G}_{0}-\mathrm{G}_{1} ; \boldsymbol{\nabla}$, ESRS2/S; $\mathbf{C ,}$ ESRS2/ $\mathrm{G}_{2}+\mathrm{M} ; \boldsymbol{\bullet}, \mathrm{ESAS} / \mathrm{G}_{0^{-}}$ $\mathrm{G}_{1} ; \boldsymbol{\Lambda}, \mathrm{ESAS} 2 / \mathrm{S} ;$ $\mathrm{ESAS} 2 / \mathrm{G}_{2}+\mathrm{M}$. with or without ESRS2

cubated with $10 \mu \mathrm{M}$ ESAS2, there were two- to fourfold decreases in EWS mRNA expression in the cells (Fig. $6 C$ ).

Effects of antisense oligonucleotide on tumor growth in nude mice. The effects of ESAS2 on tumor growth in nude mice were next examined. SK-N-MC cells were inoculated into nude mice and the tumors were grown for $7 \mathrm{~d}$, and then injection of the oligomer was started. Each mouse received a total of $500 \mathrm{nmol}$ of oligonucleotide given in five injections. The mice were killed $35 \mathrm{~d}$ after the tumor inoculation, and the subcutaneous tumors were excised and weighed. As shown in Fig. 7 and Table II, subcutaneous injections at the site of the tumor led to a significant difference in tumor growth between the antisense and control groups $(P<0.01)$. There was an approximately fourfold decrease in growth between the antisense and the control groups at day 28.

Alterations of cell cycle distribution by antisense treatment. To determine whether ESAS2 caused growth inhibition of the ES and PNET cells during any particular phase of the cell cycle, we performed FACS ${ }^{\circledR}$ analysis on propidium iodidestained cultures of SK-N-MC cells exposed to the antisense oligomer. The number of untreated and ESRS2-treated cells in the $\mathrm{G}_{0} / \mathrm{G}_{1}$ phase never exceeded $40 \%$ in 3 -d culture. Exposure of the cells to $10 \mu \mathrm{M}$ of ESAS2 resulted in a rapid increase in the number of cells accumulating in the $G_{0} / G_{1}$ phase, with a concomitant decrease in the number of cells in the $\mathrm{S}$ and $\mathrm{G}_{2}+\mathrm{M}$ phases of the cell cycle $(P<0.01)$ (Fig. 8). These results suggest that the growth of ES and PNET cells might be inhibited by antisense oligonucleotide due to a block in the cell cycle at $\mathrm{G}_{0} / \mathrm{G}_{1}$.

\section{Discussion}

ES was first reported by Ewing as a primary bone tumor (33). PNET was initially described by Stout as a soft-tissue tumor composed of small round cells characterized by the presence of rosettes (34). Close relationships between ES and PNET have been reported with reference to chromosomal abnormality (9-11), protooncogene expression $(35,36)$, and experimen- 
tal neural differentiation $(26,37,38)$. Identification of the common translocation $\mathrm{t}(11 ; 22)(\mathrm{q} 24 ; \mathrm{q} 12)$ in ES and PNET has strongly supported the hypothesis that these neoplasms have shared a common histogenesis. A recent study has investigated the molecular basis of the translocation resulting in synthesis of the EWS-Fli1 fusion gene (3). Although the function of the chimeric protein has been evaluated (13-18), the biological role of the fusion is still obscure. Recent studies have reported that ES cells transfected with antisense EWS-Fli1 cDNA expressing plasmids resulted in decreased EWS-Fli1 expression levels and in growth reduction of the cells $(19,20)$. The objective of this study was to demonstrate that EWS-Fli1 might play an important role in the growth of ES and PNET cells, and that antisense oligonucleotides against EWS-Fli1 RNA could inhibit proliferation of ES and PNET cells both in vitro and in vivo.

EWS-Fli1 mRNA was detected in all ES and PNET cell lines using standard RT-PCR. Since the expression levels seemed to be increased in cells that exhibited rapid cell growth, we established a C-PCR assay to provide a more accurate assessment of the differences. The C-PCR technique has been shown to be applicable for quantification of rare messages or small samples (39-42). In this study, the assay was reliable in the efficiency of the RT reaction, the linearity of exponential amplification of PCR, and the sample-to-sample variations between individual PCR vials. Furthermore, the use of the same primer-binding sites both in competitive template and endogenous EWS-Fli1 enabled us to obtain identical amplification efficiency. EWS-Fli1 mRNA was reproducibly quantitated in the range of 1-1,000 amol. Using this assay, we found that $E W S$-Flil expression was elevated in the cells exhibiting rapid growth. The amount of chimeric cDNA obtained from SK-N-MC, which is the fastest growing cell line, was 21.4-fold that obtained from SK-ES-1, which is the slowest growing cell line. The increase in the mRNA expression was antiparalleled by the increase in the cell population doubling time. Thus, we postulated that EWS-Fli1 might play an important role in the proliferation of ES and PNET cells. To obtain support for this notion, we applied antisense phosphorothioate oligodeoxynucleotides that inhibit the function of the chimeric RNA. Our data clearly demonstrate the pronounced effectiveness of the antisense sequences against EWS-Flil fusion RNA in the inhibition of growth of ES and PNET cells. The growth inhibition was specific to the antisense oligomers, since none of the sense or scrambled oligonucleotides exhibited the growth-inhibitory effects. The antisense oligomer also exerted the inhibitory effects on the growth of SK-N-MC tumors in nude mice. When the antisense sequences were applied, the expression levels of EWS-Fli1 mRNA and its product, EWSFli1 protein, were significantly reduced in all analyzed ES and PNET cells. Therefore, we concluded that EWS-Fli1 might be necessary for the continuous proliferation of ES and PNET cells, and that EWS-Fli1 might be a therapeutic target for inhibition of the growth of these tumors.

In this study, we used five antisense oligomers. BPAS1 and BPAS2 are complementary to the EWS-Fli1 breakpoint and specific to the fusion RNA, while ESAS1, ESAS2, and ESAS3 are complementary to the region including ATG initiation codon of EWS-Fli1. ESAS2 was the most effective in inhibiting ES and PNET cell growth. Since the sequences targeted to the initiation codon are also complementary to normal $E W S$, there is a possibility that the antisense oligomers might hybridize to
EWS RNA, as well as to EWS-Fli1 RNA. Thus, the expression of $E W S$ mRNA in various cell lines was examined. The $E W S$ mRNA could be detected not only in ES and PNET cells, but also in IMR-32 and MRC-5. Using C-PCR analysis, we found that ESAS2 also suppressed the EWS mRNA levels in all these cells. However, the growth of both IMR-32 and MRC-5 cells was not affected by the antisense oligomers. Although we could not exclude the possible implications of the decrease in the EWS mRNA in the cells, we concluded that the inhibitory effects of the antisense oligonucleotides might be mediated, at least in part, by the inhibition of the function of EWS-Fli1. This antisense approach might be useful for the inhibition of growth of other EWS-rearranged tumors including ES and PNET with $E W S$-ERG $(43,44)$ or $E W S$-ETV1 (45), malignant melanoma with $E W S$ - $A T F 1$ (46), and desmoplastic small round cell tumor with $E W S$-WT1 (47).

To obtain further evidence of the growth-related function of EWS-Fli1, we observed the effects of the antisense oligonucleotide on the modulation of cell cycle progression in EWSFli1-expressing cells. Proliferation of mammalian cells is regulated by a large number of genes whose expression or functions respond to mitogenic stimuli (48-50). Some transcriptional factors, such as c-Fos, c-Jun, and c-Myc, are known to be essential for cell cycle progression $(51,52)$. In this study, we demonstrated that inhibition of the function of EWS-Fli1 by antisense sequence resulted in the $\mathrm{G}_{0} / \mathrm{G}_{1}$ block in the cell cycle progression. In this regard, it should be noted that the enforced expression of EWS-Fli1 upregulates the activity of the $c$-myc promoter (16), and that two members of the ETS family, ETS1 and ETS2, were able to facilitate $c$-myc expression and promote cell cycle progression in mutant NIH3T3 cells arrested in the $G_{1}$ phase because there had been no induction of $c$-myc expression in response to growth factor $(53,54)$. It is therefore possible that EWS-Fli1 may activate cell division through transcriptional activation of growth-stimulating genes, such as $c-m y c$. On the other hand, a recent study revealed that expression of some genes might be suppressed when the EWSFli1 gene was transfected into fibroblasts (18). This suggests another possible mechanism whereby EWS-Fli1 might repress a gene(s) that suppresses cell growth just as tumor-suppresser genes do. Identification of the EWS-Fli1 target genes, which directly contribute to the dysregulation of cell growth, is necessary to further analyze the mechanism of carcinogenesis caused by this protein.

Recent advances in adjuvant chemotherapy have improved the prognosis of ES and PNET. However, 5-yr survival rates of the recurrent and/or metastatic cases still remain below $10 \%$ (55-58). We observed a significant growth inhibition of ES and PNET cells by inhibiting the expression of EWS-Flil chimeric RNA. It is likely that the neoplastic growth of ES and PNET depends upon the continued expression of the EWS-Fli1 gene. Thus, the chimeric RNA could be used as a target to specifically inhibit the growth of the sarcoma cells. A promising outcome of this research would be the possibility of treating ES and PNET with the specific antisense oligonucleotide.

\section{Acknowledgments}

We are most grateful to Drs. Hynda K. Kleinman, Yoshihiko Yamada, and Yoichi Sugioka for their helpful advice and discussion. We thank Dr. Sinya Oda for FACS ${ }^{\circledR}$ analysis. 
This work was supported in part by a Grant-in-Aid for General Scientific Research (06671462) from the Ministry of Education, Science, Sports and Culture (Japan), a Grant-in-Aid for Cancer Research (5-33) from the Ministry of Health and Welfare (Japan), and the Uehara Memorial Foundation.

\section{References}

1. Rabbitts, T.H. 1994. Chromosomal translocations in human cancer. $\mathrm{Na}$ ture (Lond.). 372:143-149.

2. Sreekantaiah, C., M. Ladanyi, E. Rodriguez, and R.S.K. Chaganti. 1994. Chromosomal aberrations in soft tissue tumors. Am. J. Pathol. 144:1121-1134.

3. Delattre, O., J. Zucman, B. Plougastel, C. Desmaze, T. Melot, M. Peter, H. Kovar, I. Joubert, P.D. Jong, G. Rouleau, A. Aurias, and G. Thomas. 1992. Gene fusion with an ETS DNA-binding domain caused by chromosome translocation in human tumors. Nature (Lond.). 359:162-165.

4. Crozat, A., P. Aman, N. Mandahl, and D. Ron. 1993. Fusion of CHOP to a novel RNA-binding protein in human myxoid liposarcoma. Nature (Lond.). 363:640-644.

5. Rabbitts, T.H., A. Forster, R. Larson, and P. Nathan. 1993. Fusion of the dominant negative transcription regulator $C H O P$ with a novel gene FUS by translocation $\mathrm{t}(12 ; 16)$ in malignant liposarcoma. Nat. Genet. 4:175-180.

6. Barr, F.G., N. Galili, J. Holick, J.A. Biegel, G. Rovera, and B.S. Emanuel. 1993. Rearrangement of the PAX3 paired box gene in the pediatric solid tumor alveolar rhabdomyosarcoma. Nat. Genet. 3:113-117.

7. Galili, N., R.J. Davis, W.J. Fredericks, S. Mukhopadhyay, F.J. Rauscher III, B.S. Emanuel, G. Rovera, and F.G. Barr. 1993. Fusion of a fork head domain gene to PAX3 in the solid tumor alveolar rhabdomyosarcoma. Nat. Genet. $5: 230-235$.

8. Clark, J., P.J. Rocques, A.J. Crew, S. Gill, J. Shipley, A.M. Chan, B.A. Gusterson, and C.S. Cooper. 1994. Identification of novel genes, SYT and SSX, involved in the $\mathrm{t}(\mathrm{X} ; 18)(\mathrm{p} 11.2 ; \mathrm{q} 11.2)$ translocation found in human synovial sarcoma. Nat. Genet. 7:502-508.

9. Turc-Carel, C., I. Philip, M.P. Berger, T. Philip, and G.M. Lanoir. 1983. Chromosomal translocations in Ewing's sarcoma. N. Engl. J. Med. 309:497-498.

10. Whang-Peng, J., T.J. Triche, T. Knutsen, J. Miser, E.C. Douglass, and M.A. Israel. 1984. Chromosomal translocations in peripheral neuroepithelioma. N. Engl. J. Med. 311:584-585.

11. Whang-Peng, J., T.J. Triche, T. Knutsen, J. Miser, S. Kao-Shan, S. Tsai, and M.A. Israel. 1986. Cytogenetic characterization of selected small round cell tumors of childhood. Cancer Genet. Cytogenet. 21:185-208.

12. Zhang, L., V. Lemarchandel, P.H. Romeo, Y. Ben-David, P. Greer, and A. Bernstein. 1993. The FLI-1 protooncogene, involved in erythroleukemia and Ewing's sarcoma, encodes a transcriptional activator with DNA-binding specificities distinct from other Ets family members. Oncogene. 8:1621-1630.

13. May, W.A., M.L. Gishizky, S.L. Lessnick, L.B. Lunsford, B.C. Lewis, O. Delattre, J. Zucman, G. Thomas, and C.T. Denny. 1993. Ewing sarcoma 11;22 translocation produces a chimeric transcription factor that require the DNAbinding domain encoded by FLII for transformation. Proc. Natl. Acad. Sci. USA. 90:5752-5756.

14. May, W.A., S.L. Lessnick, B.S. Braun, M. Klemsz, B.C. Lewis, L.B. Lunsford, R. Hromas, and C.T. Denny. 1993. The Ewing sarcoma EWS/FLI-1 fusion gene encodes a more potent transcriptional activator and is a more powerful transforming gene than FLI-1. Mol. Cell. Biol. 13:7393-7398.

15. Ohno, T., V.N. Rao, and E.S. Reddy. 1993. EWS/Fli-1 chimeric protein is a transcriptional activator. Cancer Res. 53:5859-5863.

16. Bailly, R.A., R. Bosselut, J. Zucman, F. Cormier, O. Delattre, M. Roussel, G. Thomas, and J. Ghysdael. 1994. DNA-binding and transcriptional activation of the EWS-FLI-1 fusion protein resulting from the $\mathrm{t}(11 ; 22)$ translocation in Ewing sarcoma. Mol. Cell. Biol. 14:3230-3241.

17. Mao, X., S. Miesfeldt, H. Yang, J.M. Leiden, and C.B. Thompson. 1994. The FLI-1 and chimeric EWS-FLI-1 oncoproteins display similar DNA binding specificities. J. Biol. Chem. 269:18216-18222.

18. Braun, B.S., R. Frieden, S.L. Lessnick, W.A. May, and C.T. Denny. 1995. Identification of target genes for the Ewing's sarcoma EWS/FLI fusion protein by representational difference analysis. Mol. Cell. Biol. 15:4623-4630.

19. Ouchida, M., T. Ohno, Y. Fujimura, V.N. Rao, and E.S.P. Reddy. 1995. Loss of tumorigenicity of Ewing's sarcoma cells expressing antisense RNA to EWS-fusion transcripts. Oncogene. 11:1049-1054.

20. Kovar, H., D.N.T. Aryee, G. Jug, C. Henoecki, M. Schemper, O. Delattre, G. Thomas, and H. Gadner. 1996. EWS/FLI1 antagonists induce growth inhibition of Ewing tumor cells in vitro. Cell Growth Differ. 7:429-437.

21. Crooke, S.T. 1993. Progress toward oligonucleotide therapeutics: pharmacodynamic properties. FASEB J. 7:533-539.

22. Stein, C.A., and Y.C. Cheng. 1993. Antisense oligonucleotides as therapeutic agents-is the bullet really magical? Science (Wash. DC). 261:1004-1012.

23. Haeuptle, M., R. Frank, and B. Dobberstein. 1986. Translation arrest by oligodeoxynucleotides complementary to mRNA coding sequences yields polypeptides of predetermined length. Nucleic Acid Res. 14:1427-1448.

24. Walder, R.Y., and J.A. Walder. 1988. Role of RNase H in hybrid-arrested translation by antisense oligonucleotides. Proc. Natl. Acad. Sci. USA. 85:50115015 .

25. Munroe, S.H. 1988. Antisense RNA inhibits splicing of pre-mRNA in vitro. EMBO (Eur. Mol. Biol. Organ.) J. 7:2523-2532.

26. Tanaka, K., Y. Iwamoto, Y. Noguchi, Y. Oda, and Y. Sugioka. 1995. Establishment and characterization of a peripheral neuroepithelioma cell line in soft tissue of extremity. Lab. Invest. 72:237-248.

27. Chen, G., J.P. Jaffrezou, W.H. Fleming, G.E. Duran, and B.I. Sikic. 1994. Prevalence of multidrug resistance related to activation of the mdr-1 gene in human sarcoma mutants derived by single-step doxorubicin selection. Cancer Res. 54:4980-4987.

28. Tanaka, K., Y. Iwamoto, Y. Ito, T. Ishibashi, Y. Nakabeppu, M. Sekiguchi, and Y. Sugioka. 1995. Cyclic AMP-regulated synthesis of the tissue inhibitors of metalloproteinases suppresses the invasive potential of the human fibrosarcoma cell line HT1080. Cancer Res. 55:2927-2935.

29. Wilhelm, S.M., I.E. Collier, B.L. Marmer, A.Z. Eisen, G.A. Grant, and G.I. Goldberg. 1989. SV40-transformed human lung fibroblasts secrete a 92$\mathrm{kDa}$ type IV collagenase which is identical to that secreted by normal human macrophages. J. Biol. Chem. 264:17213-17221.

30. Sambrook, J., E.F. Fritsch, and T. Maniatis. 1989. Measurement of radioactivity in nucleic acids. In Molecular Cloning: A Laboratory Manual. C. Noran, editor. Cold Spring Harbor Press, New York. E.18-E.19.

31. Nakabeppu, Y., S. Oda, and M. Sekiguchi. 1993. Proliferative activation of quiescent Rat-1A cells by DFosB. Mol. Cell. Biol. 13:4157-4166.

32. Giovannini, M., J.A. Biegel, M. Serra, J.Y. Wang, Y.H. Wei, L. Nycum, B.S. Emanuel, and G.A. Evans. 1994. EWS-erg and EWS-Fli1 fusion transcripts in Ewing's sarcoma and primitive neuroectodermal tumors with variant translocations. J. Clin. Invest. 94:489-496.

33. Ewing, J. 1921. Diffuse endothelioma of bone. Proc. NY Pathol. Soc. 21: $17-24$.

34. Stout, A.P. 1918. A tumor of an ulnar nerve. Proc. NY Pathol. Soc. 18:212.

35. Thiele, C.J., C. McKeon, T.J. Triche, R.A. Ross, C.P. Reynolds, and M.A. Israel. 1987. Differential protooncogene expression characterizes histopathologically indistinguishable tumors of the peripheral nervous system. $J$. Clin. Invest. 80:804-811.

36. McKeon, C., C.J. Thiele, R.A. Ross, M. Kwan, T.J .Triche, J.S. Miser, and M.A. Israel. 1988. Indistinguishable patterns of proto-oncogene expression in two distinct but closely related tumors: Ewing's sarcoma and neuroepithelioma. Cancer Res. 48:4307-4311.

37. Cavazzana, A.O., J.S. Miser, J. Jefferson, and T.J. Triche. 1987. Experimental evidence for a neural origin of Ewing's sarcoma of bone. Am. J. Pathol. 127:507-518.

38. Noguera, R., T.J. Triche, S. Navarro, M. Tsokos, and A. LlombartBosch. 1992. Dynamic model of differentiation in Ewing's sarcoma cells: comparative analysis of morphologic, immunocytochemical, and oncogene expression parameters. Lab. Invest. 62:143-151.

39. Wang, A.M., M.V. Doyle, and D.F. Mark. 1989. Quantitation of mRNA by the polymerase chain reaction. Proc. Natl. Acad. Sci. USA. 86:9717-9721.

40. Gilliland, G., S. Perrin, K. Blanchard, and H.F. Bunn. 1990. Analysis of cytokines DNA and RNA: detection and quantitation by competitive polymerase chain reaction. Proc. Natl. Acad. Sci. USA. 87:2725-2729.

41. Siebert, P.D., and J.W. Larrick. 1992. Competitive PCR. Nature (Lond.). 259:557-558.

42. Peten, E.P., L.J. Striker, M.A. Carome, S.J. Elliott, C.W. Yang, and G.E. Striker. 1992. The contribution of increased collagen synthesis to human glomerulosclerosis: a quantitative analysis of a2IV collagen mRNA expression by competitive polymerase chain reaction. J. Exp. Med. 176:1571-1576.

43. Delattre, O., J. Zucman, T. Melot, X.S. Garau, J.M. Zucker, G.M. Lenoir, P.F. Ambros, D. Sheer, C. Turc-Carel, T.J. Triche et al. 1994. The Ewing family of tumors-a subgroup of small-round-cell tumors defined by specific chimeric transcripts. N. Engl. J. Med. 331:294-299.

44. Sorensen, P.H., S.L. Lessnick, D. Lopez-Terrada, X.F. Liu, T.J. Triche, and C.T. Denny. 1994. A second Ewing's sarcoma translocation, t(21;22), fuses the EWS gene to another ETS-family transcription factor, ERG. Nat. Genet. 6: $146-151$.

45. Jeon, I., J.N. Davis, B.S. Braun, J.E. Sublett, M.F. Roussel, C.T. Denny, and D.N. Shapiro. 1995. A variant Ewing's sarcoma translocation $(7 ; 22)$ fuses the EWS gene to the ETS gene ETV1. Oncogene. 10:1229-1234.

46. Zucman, J., O. Delattre, C. Desmaze, A.L. Epstein, G. Stenman, F. Speleman, C.D. Fletchers, A. Aurias, and G. Thomas. 1993. EWS and ATF-1 gene fusion induced by $\mathrm{t}(12 ; 22)$ translocation in malignant melanoma of soft parts. Nat. Genet. 4:341-345.

47. Ladanyi, M., and W. Gerald. 1994. Fusion of the EWS and WT1 genes in the desmoplastic small round cell tumor. Cancer Res. 54:2837-2840.

48. Herschman, H.R. 1991. Primary response genes induced by growth factors and tumor promoters. Annu. Rev. Biochem. 60:281-319.

49. Lau, L.F., and D. Nathans. 1991. Genes induced by serum growth factors. In Molecular Aspects of Cellular Regulation. Vol. 6. P. Cohen and J.G. Foulkes, editors. Elsevier Science, Amsterdam, Netherlands. 257-293.

50. Nathans, D., B.A. Christy, R. DuBois, A. Lanahan, L. Sanders, and Y. Nakabeppu. 1991. Transcriptional factors induced by growth-signaling agents. 
In Origins of Human Cancers: A Comprehensive Review. J. Brugge, T. Curran, E. Harlow, and F. McCormick, editors. Cold Spring Harbor Press, New York. 353-364

51. Ransone, L.J., and I.M. Verma. 1990. Nuclear protooncogenes Fos and Jun. Annu. Rev. Cell Biol. 6:539-557.

52. Kretzner, L., E.M. Blackwood, and R. Eisenman. 1992. Myc and Max protein possess distinct transcriptional activities. Nature (Lond.). 359:426-429.

53. Langer, S.L., D.M. Bortner, M.F. Roussel, C.J. Sherr, and M.C. Ostrowski. 1992. Mitogenic signaling by colony-stimulating factor 1 and ras is suppressed by the ets-2 DNA-binding domain and restored by myc overexpression. Mol. Cell. Biol. 12:5355-5362.

54. Roussel, M.F., J.N. Davis, J.L. Cleveland, J. Ghysdael, and S.W. Hiebert. 1994. Dual control of myc expression through a single DNA binding site targeted by ets family proteins and E2F1. Oncogene. 9:405-415.

55. Burdach, S., H. Jurgens, C. Peters, W. Nurnberger, C. Mauz-Korholz,
D. Korholz, M. Paulussen, H. Pape, D. Dilloo, E. Koscielniak et al. 1993. Myeloablative radiochemotherapy and hematopoietic stem-cell rescue in poorprognosis Ewing's sarcoma. J. Clin. Oncol. 11:1482-1488.

56. Frassica, F.J., D.A. Frassica, D.J. Pritchard, P.J. Schomberg, L.E. Wold, and F.H. Sim. 1993. Ewing sarcoma of the pelvis. Clinicopathological features and treatment. J. Bone Joint Surg. Am. Vol. 75:1457-1465.

57. Maygarden, S.J., F.B. Askin, G.P. Siegal, L.A. Gilula, J. Schoppe, M. Foulkes, J.M. Kissane, and M. Nesbit. 1993. Ewing sarcoma of bone in infants and toddlers. A clinicopathologic report from the Intergroup Ewing's Study. Cancer. 71:2109-2118.

58. Ladenstein, R., C. Lasset, R. Pinketon, J.M. Zucker, C. Peters, S. Burdach, N. Pardo, G. Dollorso, C. Coze, M. Buclon et al. 1995. Impact of megatherapy in children with high-risk Ewing's tumours in complete remission: a report from the EBMT Solid Tumor Registry. Bone Marrow Transplant. 15:697705 